\title{
Bipolar Electrocoagulation Versus Intracorporeal Hemostatic Suturing for Laparoscopic Ovarian Cystectomy: Prospective Cohort Study on Effects
}

\author{
Zakia M. Ibrahim, MD, ${ }^{1}$ Hanan M. Ghoneim, MD, ${ }^{1}$ Eman A. Kishk, MD, \\ Ahmed M. Abbas, MD, ${ }^{2,3}$ Mahmoud A. Greash, MD, and Khaled A. Atwa, MD ${ }^{1}$
}

\begin{abstract}
Objective: Intracorporeal suturing is an effective hemostatic technique after laparoscopic ovarian cystectomy, but evidence of suturing superiority over electrocoagulation is lacking. This study compared the effects of bipolar electrocoagulation versus hemostatic suturing on ovarian reserve, as assessed by antral follicle count (AFC), antiMüllerian hormone (AMH), and follicle-stimulating hormone (FSH) after laparoscopic benign ovarian cystectomy.

Materials and Methods: This prospective cohort study, conducted in a tertiary-care university hospital, enrolled 50 patients with benign ovarian cysts. The patients were divided into 2 groups: (1) intracorporeal suturing and (2) bipolar electrocoagulation. Serial ultrasounds and blood samples for AMH and FSH were assessed preoperatively; then at 3- and 6-month follow-ups.

Results: There was a statistically significant reduction in AMH $(p<0.001)$ at the 6-month follow up from baseline (preoperative) in patients managed with bipolar electrocoagulation $(-0.49 \pm 0.28 \mathrm{ng} / \mathrm{mL}$ ), compared to those who had hemostatic sutures $(-0.25 \pm 0.36 \mathrm{ng} / \mathrm{mL})$. The difference in FSH was also significantly higher in the bipolar-electrocoagulation group than in the hemostatic-sutures group $(0.68 \pm 0.49 \mathrm{mIU} / \mathrm{mL}$ versus $0.39 \pm 0.81 \mathrm{mIU} / \mathrm{mL} ; p<0.001)$. However, there was a statistically significant increase in AFC in the hemostaticsutures group, compared to the bipolar-electrocoagulation group $(2.64 \pm 1.52$ versus $1.8 \pm 2.27 ; p=0.03)$.

Conclusions: Intracorporeal hemostatic suturing is better than electrocauterization for patients undergoing laparoscopic benign ovarian cystectomy, with fewer deleterious effects on ovarian reserve markers. (J GYNECOL SURG 36:103)
\end{abstract}

Keywords: ovarian cystectomy, ovarian reserve, laparoscopy, intracorporeal suturing, electrocoagulation

\section{Introduction}

A N OVARIAN CYST IS A SAC containing fluid that is formed within the ovaries. Almost all women of reproductive age and $\sim 18 \%$ of women of postmenopausal age have ovarian cysts. ${ }^{1,2}$ During the last 2 decades, laparoscopy became a cornerstone in the treatment of benign masses, and is now considered the "gold standard" for removal of nonmalignant ovarian cysts. ${ }^{3}$ Laparoscopic ovarian cystectomy has the advantages of smaller incisions, less pain, decreased wound-infection rates, early ambulation, and rapid recovery ${ }^{4}$; however, there are concerns about ovarian reserve in relation to the surgical and hemostatic techniques used during laparoscopic ovarian cystectomy. 5

Ovarian cystectomy is started by stripping the ovarian cyst wall, then establishing adequate hemostasis with either bipolar electrocoagulation or simple suturing of the residual ovarian parenchyma. Previous studies have suggested that surgical removal of ovarian cysts-specifically endometriomas - might have a negative effect on ovarian reserve. ${ }^{6,7}$ Although ovarian reserve and risk of premature ovarian failure have been reported after laparoscopic excision of endometriomas in infertile patients, there are limited reports on the effect of coagulation versus

\footnotetext{
${ }^{1}$ Department of Obstetrics and Gynecology, Faculty of Medicine, Suez Canal University, Ismailia, Egypt.

${ }^{2}$ Department of Obstetrics and Gynecology, Faculty of Medicine, Assiut University, Assiut, Egypt.

${ }^{3}$ Department of Obstetrics and Gynecology, Women's Health Hospital, Assiut, Egypt.
} 
suturing on ovarian reserve in women with benign ovarian cysts. $^{8}$

Use of electrocoagulation is common during laparoscopic surgery as the technique is safe and reliable. Use of the electrocoagulation for hemostasis after laparoscopic removal of ovarian cysts could be associated with more destruction of the normal ovarian tissue. ${ }^{6}$ Therefore, many medical centers have used alternative methods as hemostatic sealants for controlling ovarian-bed bleeding after cystectomy. ${ }^{9}$

Several noninvasive markers have been proposed to assess ovarian reserve, including serum levels of folliclestimulating hormone (FSH); serum anti-Müllerian hormone $(\mathrm{AMH})$; luteinizing hormone (LH); estradiol; LH/FSH ratio; inhibin B; and sonographic variables such as mean ovarian diameter, ovarian volume, antral-follicle count (AFC), and peak systolic velocity of the ovarian artery. Among these markers, the most-sensitive seem to be AMH and AFC. ${ }^{10,11}$

It has not yet been documented whether the negative effect of cyst excision on ovarian reserve is exerted through application of bipolar electrocoagulation-which might potentially destroy the oocytes-or might be partly explained from accidental removal of healthy ovarian tissue after excision of benign cysts. ${ }^{12}$

The aim of the present study, therefore, was is to compare the effects on ovarian reserve of two different hemostatic techniques-bipolar electrocoagulation and intracorporeal ovarian hemostatic sutures-during laparoscopic benign ovarian cystectomy.

\section{Materials and Methods}

This was a prospective cohort study, conducted at a tertiary-care university hospital from September 1, 2016. to August 31, 2018. The institutional ethical review board approved the study protocol. Women who met the selection criteria were invited to participate after signing informed consents.

\section{Eligible participants}

The study included women between ages 18 and 40, diagnosed with persistent benign ovarian cysts $>5 \mathrm{~cm}$, who were scheduled to undergo laparoscopic surgery after other treatments had failed. All recruited women were generally fit for surgery. All patients who presented to the outpatient clinic with persistent ovarian cysts for more than 3 months, and who met the inclusion criteria, were invited to participate in the study.

Women with previous gynecologic surgeries, family histories of premature ovarian failure, and suspicious preoperative diagnoses of nonbenign ovarian cysts were excluded. Patients who presented with clinical pictures of complicated ovarian cysts on an emergency basis were also excluded. All woman who refused to participate in the study were also excluded.

\section{Intervention}

Eligible women who gave their informed consent were allocated to one of the study groups; bipolar electrocoagulation group (group I) or intracorporeal hemostatic suturing group (group II).
One of the study investigators collected the baseline data at inclusion through a questionnaire, a complete physical examination, ultrasound testing, and laboratory tests. The following laboratory investigations were performed to determine levels of AMH, FSH, and hemoglobin ( $\mathrm{Hb}$ ). Tumor markers (to exclude malignancy, including carcinoembryonic antigen [CEA], cancer antigen-125 [CA-125], and $\alpha$-fetoprotein [AFP]) were also investigated. All investigations were performed and then repeated at day 3 of each patient's cycle.

A combined transabdominal and transvaginal ultrasound scan was performed to diagnose of the nature of each woman's ovarian cyst in addition to assessment of her AFC.

Laparoscopic ovarian cystectomy was performed for each patient under general anesthesia, using a Veress needle through a 1-cm umbilical incision, and establishing pneumoperitoneum by $\mathrm{CO}_{2}$ insufflation to maintain an intraabdominal pressure of $15 \mathrm{~mm} \mathrm{Hg}$. An umbilical $10-\mathrm{mm}$ trocar and telescope were introduced to guide insertion of 3 additional 5-mm trocars through supra-inguinal incisions. The pelvic cavity was explored, and the antimesenteric surface of the cysts was incised, using scissors and then hydro-dissection.

After identification of the cyst wall, it was detached from the ovarian cortex by traction and countertraction forces, which were applied by using atraumatic grasping forceps. Every effort was made to excise the entire cyst without spillage of contents into the peritoneal cavity. After that, cystectomy was performed cautiously, and the cyst was extruded, using impermeable endo-bags.

Bipolar cauterization, using $50 \mathrm{~W}$ current or suturing, using (Vicryl ${ }^{\circledR} 3 / 0$; polyglactin 910 ) sutures were used to control bleeding in each patient according to her allocated group. After the removal of the ovarian cyst, specimens were assessed by visual examination for any evidence of malignancy such as vegetation, necrosis, calcification, and thick septation, etc., followed by pathologic testing to confirm the diagnosis and to rule out malignancy.

Intraoperative data was recorded regarding hemostatic technique time, feasibility, and side-effects. After the surgery, each participant was observed in the postoperative ward for 24 hours.

\section{Follow up}

Patients were seen at the outpatient clinic at 3 and 6 months after the laparoscopic operations to measure serum levels of AMH and FSH and to perform ultrasound evaluations of AFC.

\section{Study outcomes}

The primary outcome was the difference between the study groups in ovarian reserve markers at 3 and 6 months postoperatively versus the groups' baseline results. Secondary outcomes included durations of surgery, the length of hospital stays, differences in postoperative $\mathrm{Hb}$ levels versus baseline and the rate of intraoperative complications.

\section{Statistical analysis}

Data were collected and entered into a Microsoft Access database and analyzed using a Statistical Package for Social 
Science, version 22 (SPSS Inc., Chicago, IL,). Quantitative data were presented in terms of mean \pm standard deviation (SD) then compared using a Student's $t$-test, if normally distributed, or a Mann-Whitney- $U$ test if nonnormally distributed. Qualitative variables were presented as frequency and percentage. $\chi^{2}$ or Fisher's exact tests were used for comparison between the groups, according to the normality of their data. For analysis, $p<0.05$ was considered to be significant.

\section{Results}

Of 55 patients, 2 women had histories of previous ovarian cystectomies and 3 women presented with acute abdomens that were suspicious for ovarian torsion. These 5 women were excluded from the study. Fifty women were finally included in the 2 groups ( 25 in each group).

The basal sociodemographic and clinical characteristics of both groups were comparable (Table 1). There was no statistically significant difference between both groups regarding the preoperative levels of AMH, FSH, CEA, CA125, AFP, Hb, and AFC (Table 2).

The mean surgical duration was $46.08 \pm 18.33$ minutes in group I versus $67.8 \pm 16.02$ minutes in group II, with a statistically significant difference $(p<0.001)$. The duration of hospital stay was comparable (14.16 \pm 8.92 versus $15.19 \pm 7.07$ hours in groups I and II, respectively; $p=0.24$ ). The mean postoperative $\mathrm{Hb}$ level was $10.19 \pm 0.77 \mathrm{~g} / \mathrm{dL}$ in group I versus $9.61 \pm 2.12 \mathrm{~g} / \mathrm{dL}$ in group II $(p=0.193)$. However, the mean drop of $\mathrm{Hb}$ level was significantly higher in the suturing group than in the electrocoagulation group $(-1.37 \pm 1.88$ versus $-0.52 \pm 0.45 \mathrm{~g} / \mathrm{dL}$, respectively; $p<0.001)$. None of the patients in both groups developed intra- or postoperative complications or needed postoperative blood transfusion.

Table 3 shows that there was a statistically significant difference in AMH reduction values between the groups with more AMH deficit in the electrocoagulation group $(p<0.001)$. However, there was a statistically significant difference in the FSH and AFC increment values between both groups, with more FSH in the electrocoagulation group $(p<0.001)$ and more AFC increase in the hemostaticsuturing group $(p=0.036)$.

\section{Discussion}

In the present study of patients undergoing laparoscopic benign ovarian cystectomy, intracorporeal hemostatic suturing was associated with fewer deleterious effects, at a 6month follow-up, on the ovarian reserve markers $\mathrm{AMH}$, FSH, and AFC than bipolar electrocauterization.

A difference in levels of AMH is the most important indicator of ovarian reserve change, as AMH level is the only reproducible test and is not affected by diurnal or circadian rhythms. The mean difference at 6 months was $(-0.25 \pm$ $0.36)$ in the hemostatic-suturing group versus $(-0.49 \pm 0.28)$ in the electrocauterization group $(p<0.001)$. This coincided with the results of previous studies using different approaches. ${ }^{5,13,14}$ Mohamed et al. ${ }^{5}$ used laparotomic hemostatic sutures, $\mathrm{Li}$ et al. ${ }^{13}$ reported significant increases in AMH levels in a suturing group with endometriotic cysts, and Ferrero et al. ${ }^{14}$ reported similar results after stripping bilateral endometriomas. Yet, Sahin et al. ${ }^{15}$ reported no significant difference between preoperative and postoperative AMH levels $(p=0.165)$ in a hemostatic-suturing group and lower postoperative AMH levels in a bipolarelectrocoagulation group $(p=0.028)$.

Takashima et al. ${ }^{16}$ reported contradictory results, the suturing technique had a more damaging effect on ovarian reserve than electrocoagulation. However, the follow-up time was only 3 months and the sample size was only 44 patients. These researchers considered the ischemic effect of the sutures on the nearby follicles as the reason for that study'sresults. ${ }^{16}$

In the current study; serum FSH increased 3 and 6 months postoperatively in the bipolar electrocauterization group more than the in hemostatic-suturing group, which could indicate more loss of ovarian reserve in the former group. The current results agreed with those of Özgönen et al.. ${ }^{17}$

Table 1. Sociodemographic and Clinical Characteristics Among Both Groups

\begin{tabular}{|c|c|c|c|}
\hline Variables & $\begin{array}{l}\text { Bipolar-electrocoagulation } \\
\quad \text { group }(\mathrm{n}=25)\end{array}$ & $\begin{array}{l}\text { Intracorporeal hemostatic-suturing } \\
\text { group }(\mathrm{n}=25)\end{array}$ & $\mathrm{p}$-Value \\
\hline $\begin{array}{l}\text { Age }(\mathrm{yrs}), \text { mean } \pm \text { SD } \\
\text { Residence, } n(\%)\end{array}$ & $28.36 \pm 5.29$ & $30.84 \pm 5.91$ & 0.125 \\
\hline $\begin{array}{l}\text { Urban } \\
\text { Rural }\end{array}$ & $\begin{array}{l}11(44) \\
14(56)\end{array}$ & $\begin{array}{l}11(44) \\
14(56)\end{array}$ & 0.99 \\
\hline $\begin{array}{l}\text { BMI, mean } \pm \text { SD } \\
\text { Menses regularity, } n(\%)\end{array}$ & $28.8 \pm 3.96$ & $28.12 \pm 4.29$ & 0.564 \\
\hline $\begin{array}{l}\text { No } \\
\text { Yes }\end{array}$ & $\begin{array}{r}4(16) \\
21(84)\end{array}$ & $\begin{array}{c}2(8) \\
23(92)\end{array}$ & 0.667 \\
\hline $\begin{array}{l}\text { Parity, } \boldsymbol{n}(\%) \\
\quad 0 \\
1 \\
2 \\
\geq 3\end{array}$ & $\begin{array}{l}20(80) \\
2(8) \\
1(4) \\
2(8)\end{array}$ & $\begin{aligned} & 12(48) \\
& 4(16) \\
& 3(12) \\
& 6(24)\end{aligned}$ & 0.129 \\
\hline $\begin{array}{l}\text { Previous miscarriage, } \boldsymbol{n}( \\
\text { No } \\
\text { Yes }\end{array}$ & $\begin{aligned} 23 & (92) \\
2 & (8)\end{aligned}$ & $\begin{aligned} 25 & (100) \\
0 & (0)\end{aligned}$ & 0.149 \\
\hline
\end{tabular}

yrs, years; SD, standard deviation; BMI, body mass index. 
Table 2. Preoperative Investigations Among Both Groups

\begin{tabular}{lccc}
\hline Variables & $\begin{array}{c}\text { Bipolar electrocoagulation } \\
\text { group }(\mathrm{n}=25)\end{array}$ & $\begin{array}{c}\text { Intracorporeal hemostatic-suturing } \\
\text { group }(\mathrm{n}=25)\end{array}$ & $\mathrm{p}$-Value \\
\hline $\mathrm{AMH}(\mathrm{ng} / \mathrm{mL})$ & $2.78 \pm 1.77$ & $2.8 \pm 1.6$ & 0.955 \\
$\mathrm{FSH}(\mathrm{mIU} / \mathrm{mL})$ & $4.7 \pm 1.5$ & $5.18 \pm 1.79$ & 0.512 \\
$\mathrm{CA}-125(\mathrm{U} / \mathrm{mL}$ & $37.24 \pm 34.09$ & $29.43 \pm 21.78$ & 0.782 \\
$\mathrm{CEA}(\mathrm{ng} / \mathrm{mL})$ & $1.42 \pm 0.83$ & $1.39 \pm 0.96$ & 0.113 \\
$\mathrm{AFP}(\mathrm{ng} / \mathrm{mL})$ & $3.6 \pm 1.31$ & $3.64 \pm 1.21$ & 0.353 \\
$\mathrm{Hb}(\mathrm{g} / \mathrm{dL})$ & $10.71 \pm 0.74$ & $10.98 \pm 0.95$ & 0.309 \\
AFC & $5.24 \pm 1.56$ & $5.88 \pm 2.2$ & 0.282 \\
\hline
\end{tabular}

All data are presented as mean \pm standard deviation.

$\mathrm{AMH}$, anti-Müllerian hormone; FSH, follicle-stimulating hormone; CA-125, cancer antigen-125, CEA, carcinoembryonic antigen; AFP, $\alpha$-fetoprotein; $\mathrm{Hb}$, hemoglobin; AFC; antral follicle count.

who found an increase in serum FSH from $(5.72 \pm 1.78$ to $6.96 \pm 1.86)$ in an electrocauterization group versus $(5.90 \pm$ 2.03 to $6.38 \pm 1.92$ ) in a suture group 3 months postoperatively. However, these researchers did not study AMH before and after the operation. ${ }^{17}$ Additionally, Fedele et al. ${ }^{18}$ reported that bipolar electrocoagulation caused an elevation of FSH at 3, 6, and 12 months in women who had single ovaries with only endometriotic cysts.

$\mathrm{Li}$ et al. $^{9}$ included 191 women and reported that ovarian reserve (evaluated by basal $\mathrm{FSH}, \mathrm{AFC}$, and stromal vascular Doppler) decreased significantly in patients managed by electrocoagulation, compared to patients managed by ovarian suture. In addition, FSH levels at postoperative 1st, 3rd, 6 th, and 12th months were significantly increased after ovarian cystectomy. However, these findings were on patients with bilateral ovarian cysts only and there was increased serum FSH at only the 1st month in a unilateral cyst group managed by bipolar diathermy.

The last marker of ovarian reserve tests studied in the current study was AFC, which is another sensitive marker of ovarian reserve, based on the histologic assessment of the primordial follicle pool. However, determining the preop- erative AFC in a cystic ovary is technically challenging and inaccurate, making it difficult to determine the differences between pre- and postoperative AFC.

In the current study; AFC was increased or stationary at 3 months postoperatively, with no further increase at the 6month assessment in both groups. Moreover, AFC increased more in the hemostatic suturing-group, which indicated fewer effects of the suturing technique on the patients ovarian reserves.

The same results were documented by Coric et al., ${ }^{19}$ as there was a significantly higher median AFC in sutured ovaries than electrocoagulated ovaries. Sahin et al. ${ }^{15}$ found that AFC levels were significantly lower in an electrocauterization group than in a hemostatic-suture group throughout that study's follow-up time; however, AFC did not worsen in either group during that follow-up time. Therefore, bipolar electrocoagulation negatively affected the AFC. A number of studies that confirmed this observation at 3,6 , and 12 months as the previously mentioned studies such as Li et al. in $2009^{9}$, and Li et al. in 2013. ${ }^{13}$

With respect to the secondary objectives of the current study; the duration of surgery was significantly longer in the

Table 3. Ovarian Reserve Markers at Follow-Up Visits

\begin{tabular}{|c|c|c|c|}
\hline Variables & $\begin{array}{l}\text { Bipolar-electrocoagulation } \\
\text { group }(\mathrm{n}=25)\end{array}$ & $\begin{array}{l}\text { Intracorporeal hemostatic-suturing } \\
\text { group }(\mathrm{n}=25)\end{array}$ & $\mathrm{p}$-Value \\
\hline \multicolumn{4}{|l|}{ AMH (ng/mL) } \\
\hline Pre-operative & $2.78 \pm 1.77$ & $2.8 \pm 1.6$ & 0.955 \\
\hline 3 mo postoperative & $2.36 \pm 1.58$ & $2.57 \pm 1.43$ & 0.534 \\
\hline 6 mo postoperative & $2.28 \pm 1.57$ & $2.55 \pm 1.43$ & 0.473 \\
\hline AMH difference & $-0.49 \pm 0.28$ & $-0.25 \pm 0.36$ & $<0.001 *$ \\
\hline \multicolumn{4}{|l|}{ FSH (mIU/mL) } \\
\hline Preoperative & $4.7 \pm 1.5$ & $5.18 \pm 1.79$ & 0.512 \\
\hline 3 mo postoperative & $5.19 \pm 1.62$ & $5.55 \pm 1.99$ & 0.805 \\
\hline 6 mo postoperative & $5.37 \pm 1.75$ & $5.57 \pm 2.02$ & 0.915 \\
\hline FSH difference & $0.68 \pm 0.49$ & $0.39 \pm 0.81$ & $<0.000 *$ \\
\hline \multicolumn{4}{|l|}{ AFC } \\
\hline Preoperative & $5.24 \pm 1.56$ & $5.88 \pm 2.2$ & 0.282 \\
\hline 3 mo postoperative & $6.44 \pm 1.94$ & $7.48 \pm 2.65$ & 0.120 \\
\hline 6 mo postoperative & $7.04 \pm 2.62$ & $8.52 \pm 3.07$ & 0.074 \\
\hline AFC difference & $1.8 \pm 2.27$ & $2.64 \pm 1.52$ & $0.036 *$ \\
\hline
\end{tabular}

All data are presented as mean \pm standard deviation.

*Statistically significant difference.

$\mathrm{AMH}$, anti-Müllerian hormone; mo, months; FSH, follicle-stimulating hormone; AFC, antral follicle count. 
hemostatic-suturing group in the electrocauterization group $(67.8 \pm 16.02$ versus $46.08 \pm 18.33$ minutes, respectively). This could be explained by more time consumed for performing a suture intracorporeally by laparoscopy. This was also noticed by Mahmoud et al., ${ }^{20}$ as the duration of surgery in a suturing group was $\sim 91.2$ minutes, while, in an electrocoagulation group the surgery duration was $\sim 70$ minutes $(p<0.001)$. Sahin et al. ${ }^{15}$ also reported more operative time for the suturing technique with electrocauterization group $(84.6 \pm 25.1$ versus $69.8 \pm 25.2$ minutes, respectively), which was statistically significant.

In the current study, the postoperative $\mathrm{Hb}$ difference in $\mathrm{g} / \mathrm{dL}$ was $-1.37 \pm 1.88$ in the hemostatic-suturing group versus $-0.52 \pm 0.45$ in the electrocauterization group. This was mainly due to the longer time of hemostasis needed for the suturing technique, resulting in more blood loss and more $\mathrm{Hb}$ loss. However, Mahmoud et al. ${ }^{20}$ reported no difference between postoperative $\mathrm{Hb}$ and hematocrit values in both of that study's groups. Additionally, Sahin et al. ${ }^{15}$ reported no changes in postoperative $\mathrm{Hb}$ in both groups in that other study.

The mean hospital stays (in hours) in the hemostaticsuturing group was more than that of the electrocauterization group $(16.16 \pm 7.07$ versus $14.16 \pm 8.92)$ with no statistical difference between the groups. Mahmoud et al. ${ }^{20}$ reported the same results, as mean hospital stay was 9.1hours in a suture group, while it was only 7.1 hours in an electrocoagulation group. Yet, the hospital stay was statistically higher in the suture group, and the $p$-value was $<0.001$.

In the current study none of the patients suffered from postoperative ovarian hematomas; however, this occurred in the study of Mahmoud et al., ${ }^{20}$ as 1 patient in that study's suture group developed an ovarian hematoma that was detected on follow-up and treated conservatively.

\section{Conclusions}

The current study suggests that intracorporeal hemostatic suturing is an effective and safe option for patients who are undergoing laparoscopic ovarian cystectomy for benign ovarian cysts. Additionally, suturing is more beneficial to women with benign ovarian cysts than electrocauterization, according to ovarian-reserve markers. However, further follow-up data are needed to establish the long-term effect of this suturing on ovarian reserve.

\section{Author Disclosure Statement}

No financial conflicts of interest exist.

\section{Funding Information}

No funding was received for this article.

\section{References}

1. Valentin L, Skoog L, Epstein E. Frequency and type of adnexal lesions in autopsy material from postmenopausal women: Ultrasound study with histological correlation. Ultrasound Obstet Gynecol 2003;22:284.

2. Bottomley C, Bourne T. Diagnosis and management of ovarian cyst accidents. Best Pract Res Clin Obstet Gynaecol 2009;23:711.
3. Brun JL, Fritel X, Aubard Y, et al. Management of presumed benign ovarian tumors: Updated French guidelines. Eur J Obstet Gynecol Reprod Biol 2014;183:52.

4. Donnez J, Nisolle M, Gillerot S, Anaf V, Clerckx-Braun F, Casanas-Roux F. Ovarian endometrial cysts: The role of gonadotropin-releasing hormone agonist and/or drainage. Fertil Steril 1994;62:63.

5. Mohamed ML, Nouh AA, El-Behery MM, Mansour SA. Effect on ovarian reserve of laparoscopic bipolar electrocoagulation versus laparotomic hemostatic sutures during unilateral ovarian cystectomy. Int J Gynecol Obstet 2011; 114:69.

6. Celik HG, Dogan E, Okyay E, et al. Effect of laparoscopic excision of endometriomas on ovarian reserve: Serial changes in the serum antiMüllerian hormone levels. Fertil Steril 2012;97:1472.

7. Somigliana E, Berlanda N, Benaglia L, Viganò P, Vercellini P, Fedele L. Surgical excision of endometriomas and ovarian reserve: A systematic review on serum antiMüllerian hormone level modifications. Fertil Steril 2012; 98:1531.

8. Canis M, Pouly JL, Tamburro S, Mage G, Wattiez A, Bruhat MA. Ovarian response during IVF-embryo transfer cycles after laparoscopic ovarian cystectomy for endometriotic cysts of $>3 \mathrm{~cm}$ in diameter. Hum Reprod 2001; $16: 2583$.

9. Li CZ, Liu B, Wen ZQ, Sun Q. The impact of electrocoagulation on ovarian reserve after laparoscopic excision of ovarian cysts: A prospective clinical study of 191 patients. Fertil Steril 2009;92:1428.

10. Lukaszuk K, Kunicki M, Liss J, Lukaszuk M, Jakiel G. Use of ovarian reserve parameters for predicting live births in women undergoing in vitro fertilization. Eur J Obstet Gynecol Reprod Biol 2013;168:173.

11. Vural B, Cakiroglu Y, Vural F, Filiz S. Hormonal and functional biomarkers in ovarian response. Arch Gynecol Obstet 2014;289:1355.

12. Alborzi S, Foroughinia L, Kumar PV, Asadi N, Alborzi S. A comparison of histopathologic findings of ovarian tissue inadvertently excised with endometrioma and other kinds of benign ovarian cyst [sic] in patients undergoing laparoscopy versus laparotomy. Fertil Steril 2009;92: 2004.

13. Li CZ, Wei DY, Wang F, Wang HQ, Yang CR. Impact on ovarian reserve function by different homostasis methods during laparoscopic cystectomy in treatment of ovarian endometrioma [in Chinese]. Zhonghua Fu Chan Ke Za Zhi 2013;48:11.

14. Ferrero S, Venturini PL, Gillott DJ, Remorgida V, Maggiore UL. Hemostasis by bipolar coagulation versus suture after surgical stripping of bilateral ovarian endometriomas: A randomized controlled trial. J Minim Invasive Gynecol 2012;19:722.

15. Sahin C, Akdemir A, Ergenoglu AM, et al. Which should be the preferred technique during laparoscopic ovarian cystectomy? Hemostatic sutures or bipolar electrocoagulation? A randomized controlled prospective study of longterm ovarian reserve. Reprod Sci 2017;24:393.

16. Takashima A, Takeshita N, Otaka K, Kinoshita T. Effects of bipolar electrocoagulation versus suture after laparoscopic excision of ovarian endometrioma on the ovarian reserve and outcome of in vitro fertilization. J Obstet Gynaecol Res 2013;39:1246. 
17. Özgönen H, Erdemoglu E, Günyeli İ, Güney M, Mungan T. Comparison of the effects of laparoscopic bipolar electrocoagulation and intracorporeal suture application to ovarian reserve in benign ovarian cysts. Arch Gynecol Obstet 2013; 287:729.

18. Fedele L, Bianchi S, Zanconato G, Bergamini V, Berlanda N. Bipolar electrocoagulation versus suture of solitary ovary after laparoscopic excision of ovarian endometriomas. J Am Assoc Gynecol Laparosc 2004;11:344.

19. Coric M, Barisic D, Pavicic D, Karadza M, Banovic M. Electrocoagulation versus suture after laparoscopic stripping of ovarian endometriomas assessed by antral follicle count: Preliminary results of randomized clinical trial. Arch Gynecol Obstet 2011;283:373.
20. Mahmoud TH, El-Agwany AS. Safety of hemostatic sutures for hemostasis of the ovarian bed after endometriotic ovarian cystectomy. Indian J Gynecol Oncol 2016; $14: 36$.

Address correspondence to: Ahmed M. Abbas, MD Department of Obstetrics and Gynecology, Women's Health Hospital Al-Azhar Street 71511 Assiut Egypt

E-mail: bmr90@hotmail.com 